\title{
Diabetes mellitus y sus diferentes manifestaciones dermatológicas. Revisión de la literatura
}

\author{
Diana C. Calderón C. ${ }^{1}$, Alejandra Rivera ${ }^{2}$, Adriana Medina ${ }^{3}$
}

${ }^{1}$ Médica Residente de Dermatología. Fundación Universitaria de Ciencias de la Salud. Servicio dermatología. Hospital de San José

${ }^{2}$ Médica internista, Residente endocrinología, Fundación

Universitaria de Ciencias de la Salud. Hospital de San José

${ }^{3}$ Médica internista, Endocrinóloga, servicio de endocrinología

Hospital de San José. Instructora Asociada, Fundación

Universitaria de Ciencias de la Salud, Bogotá DC, Colombia.

Dirección de correspondencia: Diana Carolina Calderón

Av cra 58 N 169 a-25. 3178945208

dianaca1409@hotmail.com

Los autores no declaran conflicto de interés

Fecha de recepción: 17/03/2017

Fecha de aceptación: 01/08/2017

\section{Caso clínico}

$\mathrm{S}$ e presenta el caso de una paciente de 45 años de edad que consulta al servicio de dermatología por aparición de lesión en miembro inferior izquierdo de crecimiento progresivo, ocasionalmente dolorosa. Como antecedentes importantes presenta diabetes mellitus tipo 2 mal controlada, hipertensión arterial crónica, obesidad grado III e hipotiroidismo en suplencia. En manejo actual con metformina 2 veces día, insulina glargina 12 unidades día, losartán 50 mg día y levotiroxina $50 \mathrm{mcg}$ al día.

$\mathrm{Al}$ examen físico se evidenciaba una placa de bordes bien definidos con centro atrófico de borde eritematoso con centro pálido mal definida con múltiples vasos en su superficie, que ocupaba el 70\% de la región anterior del miembro inferior izquierdo (figura 1).

Figura 1. Placa de borde eritematoso con centro pálido mal definida con múltiples vasos en su superficie, que ocupa el $\mathbf{7 0 \%}$ de la región anterior del miembro inferior izquierdo

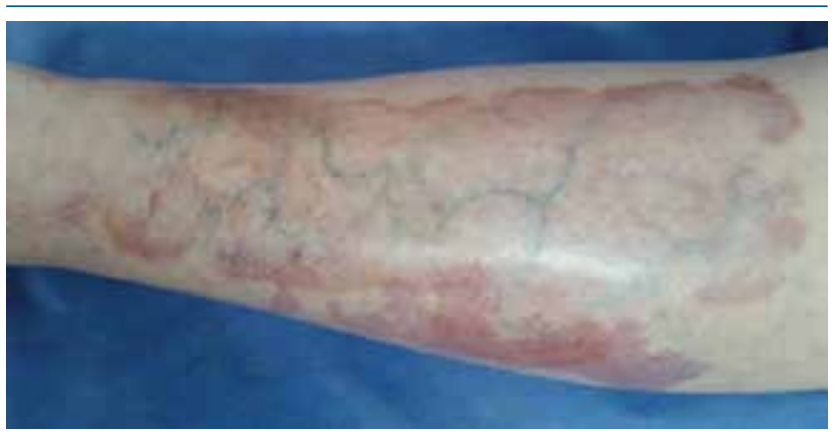

Los exámenes de laboratorio mostraban glucemia central en $278 \mathrm{mg} / \mathrm{dl}$, hemoglobina glucosilada en 9,8\%. Se realiza biopsia de piel, encontrando a nivel de la dermis colágeno necrobiótico y formación de granulomas con empalizada e infiltrado intersticial linfohistiocitario con algunas células gigantes. Se hace diagnóstico de necrobiosis lipoidica y se da manejo con fototerapia PUVA 3 veces a la semana hasta aclaramiento total de la lesión.

\section{Introducción}

La diabetes mellitus (DM) se define como un desorden metabólico, resultante de un defecto en la secreción y/o acción de la insulina, generando un estado de hiperglucemia crónico y una alteración en el metabolismo de los carbohidratos, grasas y proteínas, que resulta de múltiples etiologías $^{(1)}$. La prevalencia de diabetes va en aumento según la Organización Mundial de la Salud (OMS). En el 2014 se encontraron 422 millones de adultos en todo el mundo con diabetes, frente a los 108 millones de $1980^{(2)}$. En Latinoamérica se calcula alrededor de 15 millones de personas con diabetes mellitus y se espera que esto incremente a 20 millones en diez años. En Colombia, según un reporte de la Organización Panamericana de la Salud, existe una prevalencia del 7,5\% en la población general ${ }^{(1)}$.

Las complicaciones se producen a nivel macrovascular (ateroesclerosis) y microvascular tales como retinopatía neuropatía y nefropatía; sin embargo, existe la posibilidad de un 30-91\% de presentar al menos una complicación dermatológica durante el curso crónico de la enfermedad, según estudios reportados ${ }^{(3,4)}$. Estas manifestaciones cutáneas varían en severidad y prevalencia; muchas de estas manifestaciones son más prevalentes en pacientes con diabetes mellitus tipo 1 que en tipo 2 y muchas de ellas no son específicas de la enfermedad por lo cual se han dividido en 2 categorías: manifestaciones específicas y manifestaciones inespecíficas (infecciones -reacciones a medicamentos- complicaciones vasculares) (tabla 1$)^{(5)}$.

En esta revisión haremos énfasis en las manifestaciones específicas y algunas de las inespecíficas más relevantes, enfocándonos en la presentación clínica, la epidemiología, las causas y el tratamiento. 
Tabla 1. Manifestaciones cutáneas por categorías

\begin{tabular}{|c|c|}
\hline $\begin{array}{c}\text { Manifestaciones } \\
\text { especificas }\end{array}$ & $\begin{array}{l}\text { Manifestaciones } \\
\text { inespecificas }\end{array}$ \\
\hline $\begin{array}{l}\text { - Necrobiosis lipoídica } \\
\text { - Granuloma anular } \\
\text { generalizado } \\
\text { - Diabetes bullosa } \\
\text { - Escleredema diabeticorum } \\
\text { - Acantosis nigricans } \\
\text { - Xantomas eruptivos } \\
\text { - Dermatosis perforante } \\
\text { adquirida } \\
\text { - Dermopatía diabética }\end{array}$ & $\begin{array}{l}\text { Condiciones asociadas } \\
\text { - Acrocordones } \\
\text { - Uñas y piel amarillas } \\
\text { - Prurito generalizado } \\
\text { - Engrosamiento piel } \\
\text { - Rubeosis facies } \\
\text { - Eritema palmar } \\
\text { - Dermatosis purpúrica } \\
\text { pigmentada } \\
\text { Manifestaciones cutáneas } \\
\text { enfermedad vascular } \\
\text { - Úlceras diabéticas } \\
\text { - Pie diabético } \\
\text { Enfermedades asociadas } \\
\text { - Psoriasis } \\
\text { - Vitíligo } \\
\text { Infecciones } \\
\text { - Bacterianas } \\
\text { - Hongos } \\
\text { Complicaciones a uso de } \\
\text { terapia antidiabética } \\
\text { - Reacción cutánea a insulina } \\
\text { - Reacción cutánea a } \\
\text { antidiabético oral } \\
\text { Otros } \\
\text { - Esclerosis digital } \\
\text { Contractura de Dupuytren }\end{array}$ \\
\hline
\end{tabular}

\section{Manifestaciones específicas de diabetes mellitus}

\section{Necrobiosis Lipoidica}

Es una enfermedad granulomatosa crónica, caracterizada por la degeneración del colágeno, depósito de grasa y engrosamiento de la pared endotelial ${ }^{(6)}$. Es de baja prevalencia en la población general y presenta un aumento en la incidencia en personas con antecedente de diabetes, particularmente en manejo con insulina; esta incidencia se calcula en $0,3 \%$ a $1,2 \%$ de los cuales un 14\% aparece posterior al diagnóstico, un $24 \%$, simultáneamente y un $62 \%$ precede al diagnóstico de la diabetes ${ }^{(6)}$. Tiene un predominio en el sexo femenino de 3:1, especialmente en la tercera y la cuarta década de la vida. La patogénesis de la enfermedad aún no es completamente clara; existen diferentes teorías de las cuales, pero la principal implica un daño a nivel vascular causado por el depósito de glucoproteínas en la pared de los vasos, lo que ocasiona un en- grosamiento, fibrosis y proliferación endotelial, que llevan a la oclusión de los mismos y generan hipoxia. Este mecanismo conlleva a un proceso inflamatorio y depósito de inmunocomplejos que degeneran el colágeno en la dermis profunda ${ }^{(7,8)}$. También se presenta anormalidad en la producción de colágeno ${ }^{(9)}$, depósito de inmunoglobulinas ${ }^{(10)}$, defectos en la migración de neutrófilos ${ }^{(11)} \mathrm{y}$ factores genéticos ${ }^{(12)}$.

Las lesiones pueden ser únicas o múltiples y se presentan de manera bilateral y simétrica en la región pretibial, en la mayoría de los casos, aunque pueden aparecer en cara, tronco, cuero cabelludo y miembros superiores; clínicamente se ven como pápulas o nódulos con borde eritematoso, que confluyen formando placas con área central que inicialmente es violácea y luego progresa a una coloración pardo-amarilla de aspecto seroso (figura 1). En esta área puede haber atrofia, e incluso se puede ulcerar como complicación secundaria. Puede evidenciarse también telangiectasias y exhibirse el fenómeno de Koebner. La mayoría de estas lesiones son indoloras, resultado de daño a nivel de los nervios. Sólo un 25\% son dolorosas y un 35\% se ulceran; también pueden sufrir trasformación a un carcinoma escamocelular ${ }^{(6,13)}$. Su diagnóstico es clínico; sin embargo, se confirma mediante biopsia de piel, donde se evidencia histológicamente la formación de granulomas en dos patrones: en empalizada o pseudotuberculoide, siendo el primero el que más se ha asociado a diabetes ${ }^{(14)}$.

El tratamiento se enfoca primero en optimizar el control metabólico, haciendo énfasis en un cambio en el estilo de vida, abandono del tabaco y minimización de los traumas, para disminuir el riesgo de ulceración; adicionalmente, si se cumplen todas las recomendaciones, la terapia de primera línea es por vía tópica con corticoide intralesional o inhibidores de calcineurina. Si esto no funciona, se inicia terapia de segunda línea con pentoxifilina, fototerapia (PUVA), antimaláricos o ésteres de ácido fumárico. Finalmente, en pacientes con lesiones refractarias o ulceradas, se indica manejo con micofenolato mofetil, terapia biológica con anti TNF (infliximab, etanercept, adalimumab), ciclosporina, láser de $\mathrm{CO}_{2} \mathrm{o}$ incluso colchicina. El manejo no ha sido $100 \%$ efectivo y existe un $13-19 \%$ de los casos con autorresolución a los 6 a 12 años posteriores al diagnóstico ${ }^{(14)}$.

\section{Granuloma anular generalizado}

El granuloma anular (GA) es una enfermedad granulomatosa benigna de etiología idiopática, que afecta la dermis y el tejido celular subcutáneo ${ }^{(15)}$. Presenta diferentes variantes clínicas, de las cuales las más comunes son: GA localizado, generalizado y subcutáneo; sin embargo, existen y se han reportado en la literatura otras variantes menos comunes tales como GA perforante, de células gigantes elastolítico, en parches y palmo plantar. De todas estas variantes, la que se ha evidenciado que tiene asociación con diabetes en múltiples estudios es el granuloma anular generalizado; sin embargo, su 
prevalencia es de $0,3 \%$ en la población con diabetes mellitus y de esta se reporta que el $21-77 \%$ son diabéticos tipo 2. Es más frecuente en el sexo femenino en una proporción de 2:1,y predomina en la quinta década de la vida ${ }^{(16)}$. La patogénesis de esta enfermedad aun no es conocida; existen numerosas teorías si bien la más aceptada establece que el granuloma anular es una reacción de hipersensibilidad tipo III y esto conduce a una vasculitis que, asociada con la diabetes, genera daño mi$\operatorname{crovascular}^{(17,18)}$.

Clínicamente, se evidencian múltiples pápulas eritematosas que confluyen formando placas en forma anular (forma de anillo) distribuidas en tronco, extremidades superiores o inferiores (figura 2), y presentación atípica en un 33\%. Frecuentemente no son dolorosas y ocasionalmente causan prurito. $\mathrm{Su}$ diagnóstico es clínico; sin embargo, se debe confirmar mediante biopsia de piel en la que histológicamente puede verse la formación de granulomas en patrón en empalizada o intersticial, acompañada de depósitos de mucina ${ }^{(19)}$. El tratamiento de primera línea consiste, según la guías americanas de dermatología, en aplicación de corticoides intralesionales (acetato de triamcinolona $5 \mathrm{mg} / \mathrm{ml}$ ) y fototerapia (PUVA -UVA1). En segunda línea se encuentra el manejo con antimaláricos, la terapia biológica con anti-TNF, antimetabolito (metotrexate) y retinoides orales. Se han descrito múltiples reportes de casos manejados con dapsona, doxiciclina, ciclosporina, rifampicina e inhibidores de calcineurina tópicos, con mejoría completa de las lesiones ${ }^{(18)}$.

Figura 2. Múltiples pápulas eritematosas que confluyen formando placas de aspecto numular, algunas arciformes, comprometiendo región poplítea bilateral y región axilar posterior izquierda

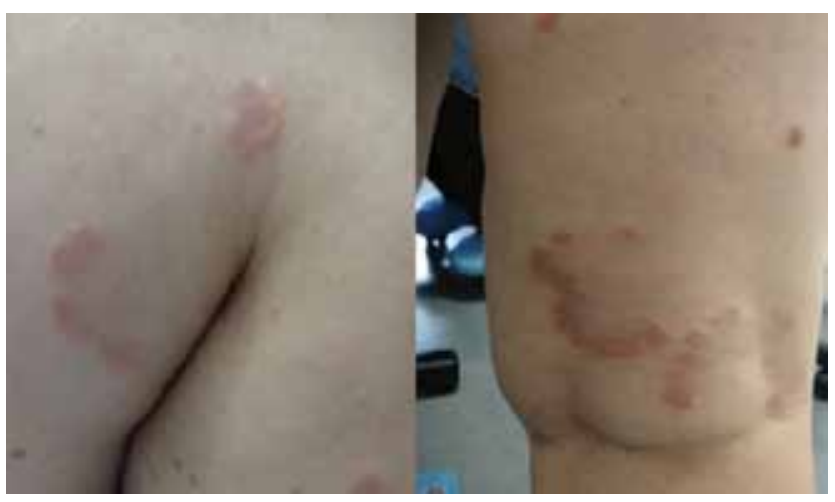

\section{Bullosis diabeticorum}

Es una condición específica de la diabetes que requiere manejo con insulina, caracterizada por la formación de ampollas tensas en regiones acrales (extremidades inferiores predominantemente y extremidades superiores con menor frecuencia), con una incidencia baja de $0,5 \%$ en USA $^{(20,21)}$ y mayor frecuencia en hombres con una relación de 2:1. La edad de aparición se encuentra entre la sexta y séptima décadas de la vida; sin embargo, se ha documentado esta condición en personas entre 30-40 años de edad. Se asocia a pacientes con complicaciones microvasculares (neuropatía, nefropatía y retinopatía) y con mal control metabólico ${ }^{(20)}$. Su etiología aún no se conoce, pero existen numerosas teorías sobre el mecanismo de formación de este desorden ampolloso, tales como traumas mínimos que inducen a la formación de ampolla, mal control metabólico (fluctuación de niveles de glucemia), microangiopatía, nefropatía, neuropatía, insuficiencia vascular y fenómeno autoinmune, además de alteración en el metabolismo del calcio. Todo esto induce a una alteración a nivel de la membrana basal, más exactamente en la lámina lúcida, dando como resultado la formación de ampollas tensas ${ }^{(5,22)}$.

Clínicamente, se caracterizan por ser ampollas tensas con diámetros que varían desde $0,5 \mathrm{~cm}$ hasta $10 \mathrm{~cm}$ con contenido viscoso estéril, que aparecen de manera espontánea en zonas acrales, asintomáticas y ocasionalmente producen prurito o sensación de quemazón. La mayoría son lesiones únicas, aunque pueden ser múltiples, distribuidas de manera asimétrica (figura 3). No existe un test confirmatorio y su diagnóstico es de exclusión. Se debe tener en cuenta otras causas de desórdenes ampollosos, tales como penfigoide ampolloso, porfiria cutánea tardía y reacciones medicamentosas, por lo que su diagnóstico se confirma mediante realización de biopsia de piel e inmunofluorescencia directa, la cual da como resultado ampolla subepidérmica en el $80 \%$ de los casos e inmunofluorescencia negativa. También se han reportado depósitos de C3 e IgM en la pared de los vasos de la dermis. Por microscopía electrónica se evidencia la ampolla subepidérmica a nivel de la lámina lúcida; sin embargo, hay casos reportados con ampollas intraepidérmicas ${ }^{(23)}$.

Figura 3. En antebrazo derecho se evidencia ampolla tensa de contenido seroso con eritema perilesional de $20 \mathrm{~mm}$ de diámetro

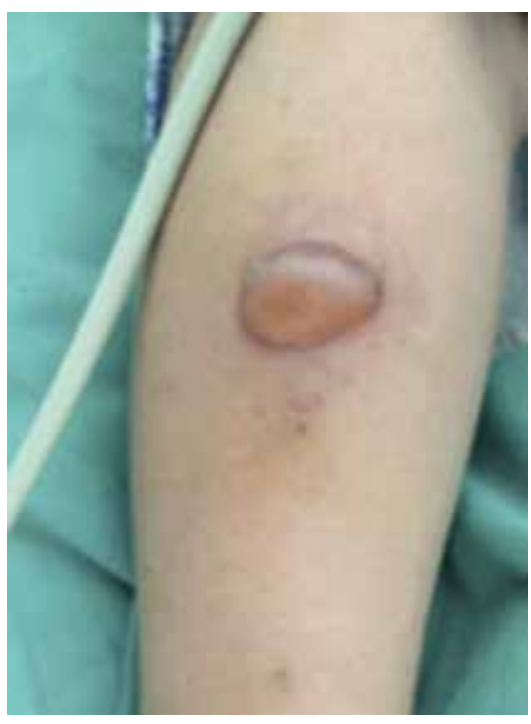


Estas ampollas resuelven espontáneamente entre 2-6 semanas; algunas pueden presentar sobreinfección (principalmente las de miembros inferiores), riesgo de osteomielitis y necrosis, por lo que se recomienda protección cutánea y manejo con antibiótico tópico para prevención de infección secundaria, aspirado de contenido de la ampolla y un adecuado control metabólico evitando episodios de hipoglucemia.

\section{Esclerema diabeticorum}

El escleredema diabeticorum es una infrecuente enfermedad del tejido conectivo que consiste en depósito de mucina en dermis y posterior endurecimiento de la piel, descrita por primera vez por Abraham Buschke en $1902^{(24)}$. Está asociada a múltiples enfermedades como gammapatía monoclonal, hiperparatiroidismo, mieloma múltiple, insulinoma maligno, artritis reumatoide, síndrome de Sjögren, infecciones estreptocóccicas y víricas, además de la diabetes ${ }^{(25)}$. Con respecto al escleredema diabeticorum, presenta una incidencia de 2,5$14 \%$ con una mayor frecuencia en hombres y una relación de 2:1. Está asociado a mal control metabólico, uso de insulina y complicaciones microvasculares ${ }^{(5,26)}$. Su patogénesis aún no se conoce y se proponen teorías, entre las cuales se encuentran: la glucosilación de fibras de colágeno con posterior degradación alterada de las mismas, el estado de hiperglucemia que estimula el fibroblasto, de tal forma que éste aumenta la síntesis de componentes de matriz extracelular tales como el colágeno, lo que lleva a desarrollar esta condición ${ }^{(26)}$.

Clínicamente se evidencia un edema duro o una piel rígida predominantemente en región cervical posterior, tronco superior y hombros. Ocasionalmente se extiende a cara, brazos, tronco anterior y abdomen. La piel puede tener aspecto de piel de naranja y algunas veces verse eritematosa. Usualmente es asintomática, pero en algunos casos causa dolor, disminuyendo la movilidad, especialmente si es a nivel de la espalda. Existen 3 patrones, según la enfermedad asociada: el primero de ellos se encuentra en mujeres de mediana edad y niños con induración en región cervicofacial secundaria a infección respiratoria aguda; el segundo patrón se ve como instauración larga e insidiosa y se asocia principalmente a gammapatías; el tercer patrón corresponde a los casos asociados a diabetes mellitus ${ }^{(27-29)}$. Su diagnóstico se realiza mediante biopsia de piel, donde se evidencia marcado engrosamiento de la dermis profunda con fibras de colágeno gruesas e infiltrado de mucina.

Aun no se ha establecido un tratamiento de elección; sin embargo, se basa primero en el control glucémico y se recomienda manejo con fototerapia tipo PUVA ${ }^{(30,31)}$ como primera elección. Además se encuentran descritos un sinnúmero de tratamientos como esteroides intralesionales, metotrexate a bajas dosis, pentoxifilina, pero aún no se llega a un consenso para el manejo de esta enfermedad.

\section{Acantosis nigricans}

La acantosis nigricans es una manifestación cutánea con múltiples etiologías, entre ellas, las más comunes son: diabetes mellitus tipo 2, obesidad y resistencia a la insulina con una prevalencia entre $7 \%-74 \%$, de predominio en mujeres de raza negra vs mujeres de raza blanca ${ }^{(32)}$. Se clasifica de dos formas: acantosis nigricans benigna y maligna. Las dos variantes tienen clínica e histología similares; no obstante, difieren en la condición asociada a la misma (tabla 2) ${ }^{(33)}$. Su fisiopatología indica que la insulina es capaz de atravesar la unión dermoepidérmica y así producir crecimiento a nivel de los queratinocitos y fibroblastos por medio del receptor del factor de crecimiento similar a la insulina tipo 1 (IGF-1Rs), lo que genera este tipo de lesiones ${ }^{(34)}$.

Tabla 2. Condiciones asociadas a Acantosis Nigricans

\begin{tabular}{l|l}
\multicolumn{1}{c|}{ Benignas } & Maligna \\
Enfermedades metabólicas & Paraneoplásicos \\
- Diabetes mellitus- resistencia & - Cáncer gástrico \\
a la insulina & \\
- Hipotiroidismo & \\
- Cirrosis biliar primaria & \\
- Enfermedad Adisson & \\
- Acromegalia & \\
- Gigantismo & \\
- Obesidad & \\
Síndromes & \\
- Síndrome ovario poliquístico & \\
- Síndrome Cushing & \\
- Síndrome de Bloom & \\
- Síndrome Marfan & \\
- Síndrome de Rud & \\
- Síndrome Down & \\
Asociada a medicamentos & \\
- Esteroides sistémicos & \\
- Ácido nicotínico & \\
- Estrógenos & \\
- Insulina & \\
- Anticonido fusídico &
\end{tabular}

Clínicamente, se caracteriza por placas hiperpigmentadas de aspecto aterciopelado o verrucoso, pardas claras a oscuras de distribución simétrica predominantemente en regiones intertriginosas y flexurales (cuello, axilas, pliegue ante cubital, pliegue inguinal y fosa poplítea), siendo más frecuente en un 99\% en el cuello y un 73\% en axilas (figura 4); usualmente son lesiones asintomáticas, aunque ocasionalmente pueden generar mal olor y prurito ${ }^{(34)}$. Su diagnóstico es clínico y sólo 
se realiza biopsia de piel cuando se requiere confirmación. En la histología se evidencia papilomatosis, hiperqueratosis e hiperpigmentación del estrato basal, debido al aumento de queratina, mas no por el aumento de la función ni el número de melanocitos ${ }^{(35)}$.

Figura 4. Placas pardas claras de aspecto aterciopelado bien definidas que ocupan el $80 \%$ de tejido celular subcutáneo en región cervical posterior y axilar
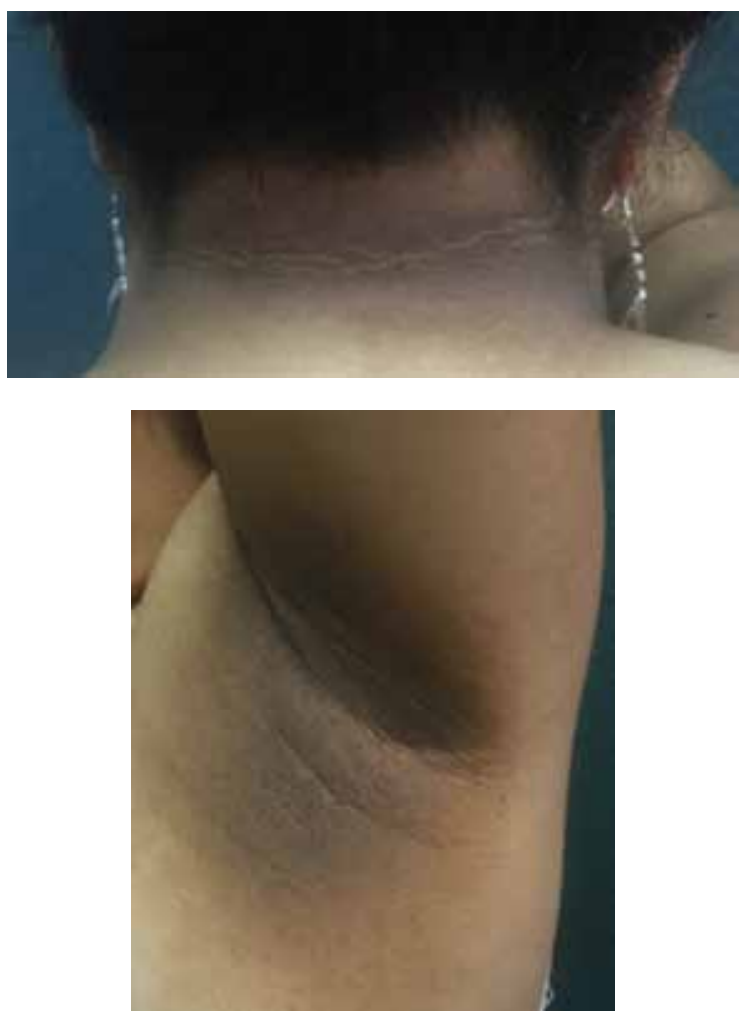

Se recomienda cambio en hábitos nutricionales, disminución de peso e incremento de la actividad física. Estas modificaciones en el estilo de vida son el pilar en el manejo de esta condición, ya que reducen la hiperinsulinemia, que es el factor principal de desarrollo de la acantosis nigricans. Se pueden utilizar medicamentos como retinoides orales que tiene como función disminuir la proliferación del queratinocito en aquellas situaciones donde la acantosis es extensa o es sintomática (mal olor, maceración o alteración en la calidad de vida) ${ }^{(36)}$.

\section{Xantomas eruptivos}

Los xantomas eruptivos en adultos están asociados con hipertrigliceridemia (nivel de triglicéridos $>2.000 \mathrm{mg} / \mathrm{dl}$ lo que implica la acumulación de quilomicrones y lipoproteínas de baja densidad (VLDL) $(37,38)$. Aproximadamente un tercio de todos los pacientes diabéticos presentan alteración en los niveles de lipoproteínas, lo cual se debe a la insulinopenia. La prevalencia de esta condición es desconocida ${ }^{(5,39)}$. La razón por la cual se producen xantomas eruptivos en pacientes diabéticos, es que la insulina es un factor estimulante de la actividad de lipoproteína lipasa y ésta juega el papel principal en el metabolismo de triglicéridos y lipoproteínas ricas en triglicéridos (quilomicrones y VLDL). En la diabetes no controlada donde hay un estado de insulinopenia existe una ausencia de actividad de lipoproteína lipasa y, como consecuencia, un aumento en lipoproteínas como VLDL y quilomicrones, generando una dislipidemia a expensas de triglicéridos y precipitando la formación de xantomas eruptivos ${ }^{(5,40)}$.

Clínicamente, se caracteriza por la presencia de múltiples pápulas de 1-4 mm xantomatosas con eritema perilesional, distribuidas principalmente en áreas extensoras (extremidades y glúteos) (figura 5). La mayoría son asintomáticas; sin embargo, ocasionalmente presentan prurito. El diagnóstico es clínico, pero se confirma mediante biopsia de piel, donde se evidencia acúmulo de macrófagos espumosos con infiltrado inflamatorio de linfocitos y neutrófilos en dermis ${ }^{(41)}$. El manejo hace énfasis en control metabólico con hábitos de vida saludable y farmacológico, con fibratos o estatinas. A nivel de las lesiones se utilizan técnicas de curetaje, láser $\mathrm{CO}_{2}$ o erbium, para removerlas con buenos resultados. Por ser una entidad poco frecuente, faltan estudios para avalar este tipo de manejos.

Figura 5. En ambos miembros inferiores se evidencian múltiples papilas xantomatosas con borde eritematoso, que ocupan el $60 \%$ de la pierna

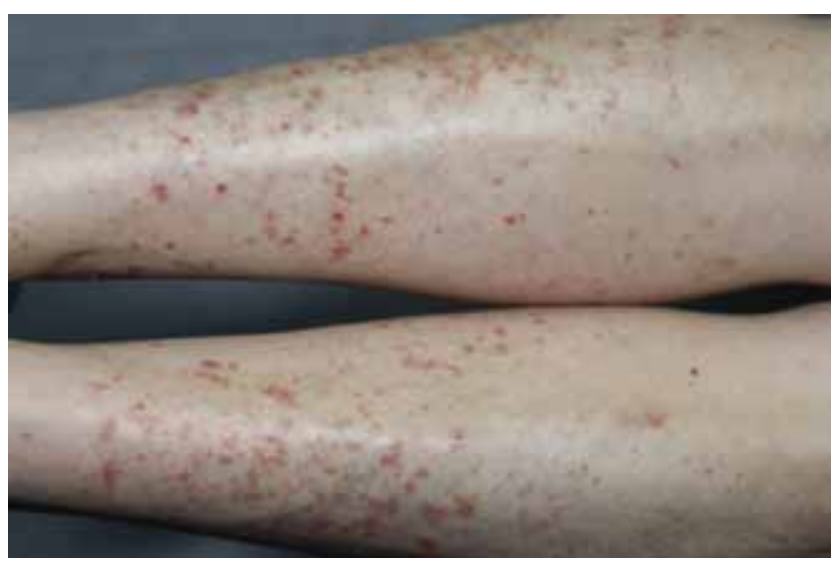

\section{Dermatopatía diabética}

La dermatopatía diabética es una manifestación cutánea frecuente, descrita desde 1960 por Hans Melin ${ }^{(42)}$, médico suizo que caracterizó estas lesiones; y en 1965, Binkley ${ }^{(43)}$ le da el nombre de dermatopatía diabética, concepto que refleja la manifestación cutánea de la microangiopatía diabética. Esta entidad tiene una incidencia del $9-55 \%$ en la población con diabetes mellitus y afecta especialmente a personas en la sexta década de la vida. Predomina en hombres con relación $2: 1^{(44-46)}$. No está clara la incidencia de la dermatopatía en pa- 
cientes con diabetes mellitus no insulinodependiente vs insulinodependiente, pero existen múltiples estudios que revelan mayor incidencia en pacientes con diabetes mellitus insulinodependiente en un $12,3 \%$, comparado con la población que no requiere insulina ${ }^{(46)}$. Lo que sí está claro es que la incidencia de la dermatopatía diabética aumenta un $52 \%$ en pacientes con complicaciones microangiopáticas (nefropatía, neuropatía o retinopatía) y en un $81 \%$ en pacientes que presentan las tres complicaciones $(\mathrm{p}<0,001)^{(47)}$. Existe además la relación entre el control glucémico y la hemoglobina glucosilada. La etiología aun es desconocida; numerosas teorías se han centrado en el trauma menor y en la microangiopatía con los cambios capilares asociados que generan estas lesiones, por lo que se sugiere que la dermatopatía diabética puede ser otra manifestación microangiopática de la diabetes mellitus ${ }^{(48)}$.

Clínicamente, se caracteriza por la presencia de múltiples placas o pápulas pardas eritematosas bien delimitadas, que evolucionan a placas atróficas con descamación fina, que pueden ulcerarse como complicación y se localizan de manera simétrica en ambos miembros inferiores, especialmente en la región pretibial, con distribución asimétrica. También pueden evidenciarse en miembros superiores y muslos (figura 6). La progresión de la dermopatía es variable y no parece estar afectada por el control metabólico. Las lesiones persisten durante 18 a 24 meses en promedio, pero pueden permanecer indefinidamente. También pueden desaparecer poco a poco, dejando atrás la hiperpigmentación residual sin atrofia ${ }^{(5,48)}$. El diagnóstico es clínico pero, en ocasiones, cuando la clínica no es específica, se realiza biopsia confirmatoria. Este examen va a evidenciar una epidermis con atrofia de las crestas epiteliales, hiperqueratosis moderada y una pigmentación variable de las células. En la dermis se observan telangiectasias, proliferación de fibroblastos, edema, microan-

Figura 6. Se evidencia de manera bilateral asimétrica placas pardo-violáceas que confluyen formando grandes placas de bordes bien definidos con descamación fina en su superficie

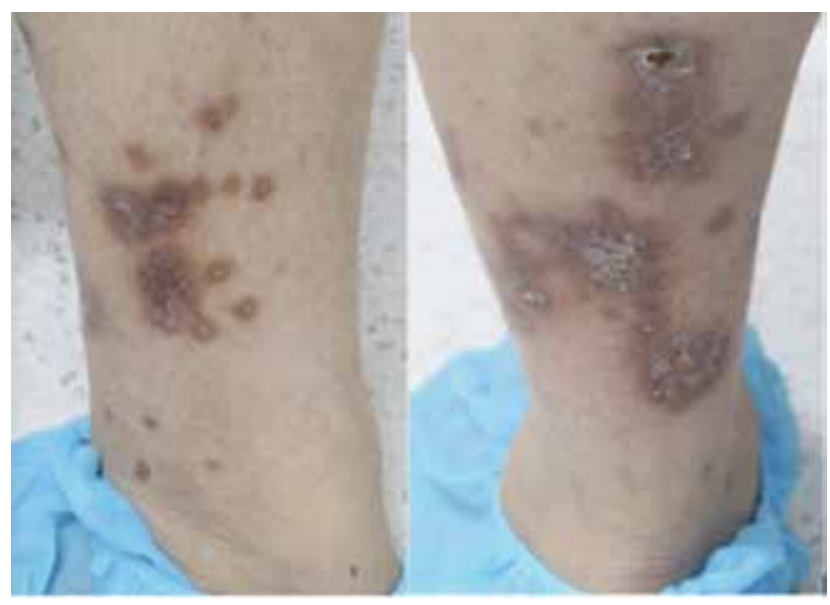

giopatía hialina, extravasación de eritrocitos y depósitos de hemosiderina ${ }^{(49,50)}$. No existe ningún manejo médico efectivo, únicamente prevención de complicaciones secundarias como infección, control glucémico y de las comorbilidades de base $^{(48,51)}$.

\section{Dermatosis perforante adquirida}

La dermatosis perforante adquirida comprende un grupo de enfermedades crónicas de la piel, que se caracterizan por la eliminación transepidérmica de material de la dermis (colágeno, fibras elásticas o tejido conjuntivo necrótico) ${ }^{(52)}$. Se clasifica según el material dérmico predominante. Se relaciona con insuficiencia renal crónica en estadios avanzados, con o sin hemodiálisis y diabetes (frecuentemente relacionada con la nefropatía diabética). Su prevalencia en pacientes en diálisis es del $10 \%$, aunque de los pacientes con insuficiencia renal, el $73 \%$ se encuentra en diálisis, y entre estos, el 50\% tiene diabetes, por lo que se calcula que la incidencia de esta dermatosis es del $13,6 \%{ }^{(53)}$. Su etiología aún no se conoce, pero numerosas teorías han concluido que una alteración metabólica causa alteración epidérmica o dérmica; que el depósito de sustancias que no se eliminan por diálisis puede ser reconocido por el sistema inmune como un cuerpo extraño, y que pueden manifestarse microtraumas como microangiopatía ${ }^{(5)}$.

Clínicamente, las lesiones son pápulas o nódulos hiperqueratósicos con tapones de queratina central, ocasionalmente umbilicados, foliculares generalizados, con patrón de distribución serpiginoso y con fenómeno de Koebner positivo. Son muy pruriginosas, causando múltiples escoriaciones (figura 7). Su diagnóstico es histológico y éste evidencia la eliminación transepidérmica del material de la dermis ${ }^{(5,54)}$. El tratamiento en el contexto de diabetes tiene tasa de efectividad baja; sin embargo, se deben evitar traumas y se debe realizar

Figura 7. Múltiples placas eritemato-violáceas, algunas hiperqueratósicas bien definidas en miembro inferior izquierdo

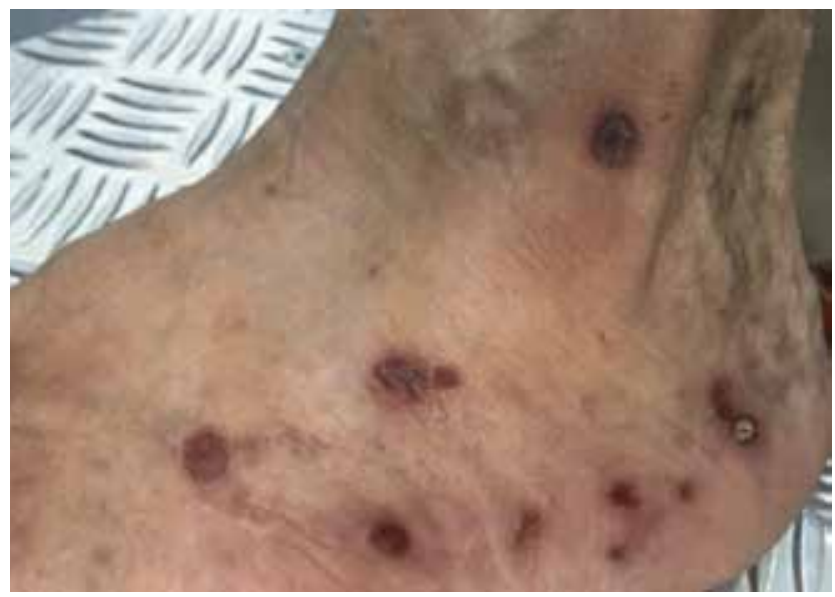


manejo sintomático del prurito. Existe la indicación de manejo con fototerapia PUVA y UVB NB con alta eficacia y manejo con queratolíticos tópicos; se han empleado corticoides intralesionales y crioterapia con mejoría del cuadro clínico ${ }^{(54)}$.

\section{Manifestaciones no específicas de diabetes mellitus}

Las manifestaciones no específicas de la diabetes mellitus se enumeran en la tabla 3.

Tabla 3. Manifestaciones no específicas de la diabetes mellitus

\begin{tabular}{|c|c|c|c|c|}
\hline $\begin{array}{l}\text { Manifestación } \\
\text { asociada }\end{array}$ & Definición & Epidemiologia & Características clínicas & Manejo \\
\hline Acrocordones & $\begin{array}{l}\text { Lesiones tumorales } \\
\text { benignas asociadas a } \\
\text { alteración de metabolismo } \\
\text { de lípidos y carbohidratos } \\
\text { (56), generando un estado } \\
\text { de hiperinsulinemia } \\
\text { y éste tiene un efecto } \\
\text { hiperproliferativo a nivel } \\
\text { del queratinocito y el } \\
\text { fibroblasto. }\end{array}$ & $\begin{array}{l}\text { Se asocia : } \\
\text { - Un } 80 \% \text { se asocia a } \\
\text { síndrome metabólico } \\
\text { (resistencia a la insulina) }^{(57)} \\
\text { - Un } 65 \%-70 \% \text { de la } \\
\text { población diabética } \\
\text { presenta acrocordones }{ }^{(58)} \\
\text { - Un } 59,1 \% \text { se asocia a } \\
\text { dislipidemia } \\
\text { - Un } 30,1 \% \text { se asocia a } \\
\text { hipertensión arterial }\end{array}$ & $\begin{array}{l}\text { Consisten en múltiples pápulas pardas } \\
\text { claras u oscuras pediculadas localizadas } \\
\text { principalmente en pliegues (cuello, } \\
\text { pliegue inframamario, axilas) y cara. } \\
\text { Asintomáticas (figura 8) }\end{array}$ & $\begin{array}{l}\text { Manejo cosmético } \\
\text { con curetaje, } \\
\text { crioterapia o } \\
\text { resección de las } \\
\text { mismas }\end{array}$ \\
\hline Piel y uñas amarillas & $\begin{array}{l}\text { Consiste en la } \\
\text { hiperpigmentación } \\
\text { amarilla en las uñas } \\
\text { y en la piel, causada } \\
\text { por productos de } \\
\text { la glucosilación no } \\
\text { enzimática ( } 2 \text { furoyl , } 2 \text {, } \\
\text { furanil y } 1 \text { h-imidazol que } \\
\text { genera el depósito de } \\
\text { pigmento amarillo a nivel } \\
\text { de la dermis }\end{array}$ & $\begin{array}{l}\text { Se asocia en un } 40 \% \text { con } \\
\text { diabetes mellitus }\end{array}$ & $\begin{array}{l}\text { Consiste en pigmentación amarilla } \\
\text { bilateral simétrica de las uñas. Y tinte } \\
\text { ictérico que no compromete las } \\
\text { escleras en la piel (figura 9) }{ }^{(62)}\end{array}$ & $\begin{array}{l}\text { Condición } \\
\text { benigna se } \\
\text { aconseja control } \\
\text { metabólico }\end{array}$ \\
\hline Rubeosis fascies & $\begin{array}{l}\text { Condición caracterizada } \\
\text { por flushing crónico } \\
\text { en cara y cuello con } \\
\text { mayor incidencia en } \\
\text { fototipos Fitzpatrick } 1 \text { y } \\
2 \text {; asociado a alteración } \\
\text { microangiopática y } \\
\text { vasodilatación venosa } \\
\text { superficial facial }\end{array}$ & $\begin{array}{l}\text { - Asociada en un } 59 \% \text { a } \\
\text { diabetes mellitus }^{(64)}\end{array}$ & $\begin{array}{l}\text { Se caracteriza por eritema facial crónico } \\
\text { en cara y cuello (figura 10) }\end{array}$ & $\begin{array}{l}\text { Condición } \\
\text { benigna, se } \\
\text { aconseja control } \\
\text { metabólico y uso } \\
\text { de fotoprotección } \\
\text { cutánea }\end{array}$ \\
\hline $\begin{array}{l}\text { Purpura } \\
\text { pigmentaria }\end{array}$ & $\begin{array}{l}\text { Es un grupo heterogéneo } \\
\text { idiopático no frecuente, } \\
\text { progresivo caracterizado } \\
\text { por una pigmentación ocre } \\
\text { en miembros inferiores }{ }^{(65)} \\
\text { causada por extravasación } \\
\text { de eritrocitos de los plexos } \\
\text { venosos superficial y } \\
\text { posterior depósito de } \\
\text { hemosiderina en la piel. }\end{array}$ & $\begin{array}{l}\text { Se asocia en un } 50 \% \\
\text { a la diabetes mellitus } \\
\text { y coexiste con la } \\
\text { dermatopatía diabética }{ }^{(66)}\end{array}$ & $\begin{array}{l}\text { Se caracteriza por máculas } \\
\text { hiperpigmentadas color pardo claro a } \\
\text { oscuro de } 0.3 \text { a } 1 \mathrm{~cm} \text { en región pretibial } \\
\text { de manera bilateral; se conocen como } \\
\text { manchas de pimienta cayena. }\end{array}$ & $\begin{array}{l}\text { Condición } \\
\text { benigna, se } \\
\text { aconseja control } \\
\text { metabólico }\end{array}$ \\
\hline
\end{tabular}

\section{Conclusiones}

Cerca del $60 \%$ de los pacientes diabéticos presentan alguna manifestación cutánea. Estos hallazgos deben interpretarse como señal de advertencia de un mal control metabólico o complicaciones microangiopáticas, tales como retinopatía, nefropatía y neuropatía; por esto, es muy importante identificar este tipo de lesiones y dar un óptimo manejo para evitar complicaciones secundarias. Se presenta una revisión completa de las manifestaciones específicas cutáneas de la diabetes mellitus, haciendo énfasis en su incidencia, la clínica y el diagnóstico de manera clara y precisa; no obstante, a pesar de numerosas investigaciones, las causas exactas de muchas complicaciones cutáneas de la diabetes aún no se conocen. Los datos demográficos varían ampliamente y van de la mano de las comorbilidades asociadas. Se debe continuar estudiando los diferentes mecanismos fisiopatológicos de la enfermedad, para así dar un mejor tratamiento y fomentar estilos de vida saludable ya que estos son los que tienen mayor impacto y pueden detener la epidemia de la diabetes mellitus y sus complicaciones. 


\section{Referencias}

1. Guías ALAD sobre el diagnóstico, control y tratamiento de la diabetes mellitus tipo 2 con medicina basada en evidencia. Revista de la ALAD. 2013.

2. www.who.int/diabetes/global-report/es/.

3. Perez MI, Kohn SR. Cutaneous manifestations of diabetes mellitus. Journal of the American Academy of Dermatology. 1994;30(4):519-31; quiz 32-4. Epub 1994/04/01.

4. Shahzad M, Al Robaee A, Al Shobaili HA, Alzolibani AA, Al Marshood AA, Al Moteri B. Skin manifestations in diabetic patients attending a diabetic clinic in the Qassim region, Saudi Arabia. Medical principles and practice: international journal of the Kuwait University, Health Science Centre. 2011;20(2):137-41. Epub 2011/01/22.

5. Murphy-Chutorian B, Han G, Cohen SR. Dermatologic manifestations of diabetes mellitus: a review. Endocrinology and metabolism clinics of North America. 2013;42(4):869-98. Epub 2013/11/30.

6. Reid SD, Ladizinski B, Lee K, Baibergenova A, Alavi A. Update on necrobiosis lipoidica: a review of etiology, diagnosis, and treatment options. Journal of the American Academy of Dermatology. 2013;69(5):783-91. Epub 2013/08/24.

7. Peyri J, Moreno A, Marcoval J. Necrobiosis lipoidica. Seminars in cutaneous medicine and surgery. 2007;26(2):87-9. Epub 2007/06/05.

8. Boateng B, Hiller D, Albrecht HP, Hornstein OP. [Cutaneous microcirculation in pretibial necrobiosis lipoidica. Comparative laser Doppler flowmetry and oxygen partial pressure determinations in patients and healthy probands]. Der Hautarzt; Zeitschrift fur Dermatologie, Venerologie, und verwandte Gebiete. 1993;44(9):581-6. Epub 1993/09/01. Kutane Mikrozirkulation bei pratibialer Nekrobiosis lipoidica. Vergleichende Laser-Doppler-Fluxmetrie und Sauerstoffpartialdruckmessungen bei Patienten und Hautgesunden.

9. Ngo B, Wigington G, Hayes K, Huerter C, Hillman B, Adler M, et al. Skin blood flow in necrobiosis lipoidica diabeticorum. International journal of dermatology. 2008;47(4):354-8. Epub 2008/04/02.

10. Oikarinen A, Mortenhumer M, Kallioinen M, Savolainen ER. Necrobiosis lipoidica: ultrastructural and biochemical demonstration of a collagen defect. The Journal of investigative dermatology. 1987;88(2):227-32. Epub 1987/02/01.

11. Ullman S, Dahl MV. Necrobiosis lipoidica. An immunofluorescence study. Archives of dermatology. 1977;113(12):1671-3. Epub 1977/12/01.

12. Roche. E VJ, Velasco. M. Familial necrobiosis lipoidica not associated with diabetes. Dermatology Online Journal. 2007;3 (13):26

13. Sibbald. C RS, Alavi. F. Necrobiosis Lipoidica Dermatol Clin. 2015;33:34360.

14. Lynch JM, Barrett TL. Collagenolytic (necrobiotic) granulomas: part II-the 'red' granulomas. Journal of cutaneous pathology. 2004;31(6):409-18 Epub 2004/06/10.

15. Thornsberry LA, English JC, 3rd. Etiology, diagnosis, and therapeutic management of granuloma annulare: an update. American journal of clinical dermatology. 2013;14(4):279-90. Epub 2013/05/23.

16. Piette EW, Rosenbach M. Granuloma annulare: Clinical and histologic variants, epidemiology, and genetics. Journal of the American Academy of Dermatology. 2016;75(3):457-65. Epub 2016/08/21.

17. Dahl MV, Ullman S, Goltz RW. Vasculitis in granuloma annulare: histopathology and direct immunofluorescence. Archives of dermatology. 1977;113(4):463-7. Epub 1977/04/01.

18. Piette EW, Rosenbach M. Granuloma annulare: Pathogenesis, disease associations and triggers, and therapeutic options. Journal of the American Academy of Dermatology. 2016;75(3):467-79. Epub 2016/08/21.

19. Chaitra V, Inchara YK, Rajalakshmi T, Antony M. Granuloma annulare - histology reconsidered. Indian journal of dermatology, venereology and leprology. 2010;76(5):568-9. Epub 2010/09/10.

20. Larsen K, Jensen T, Karlsmark T, Holstein PE. Incidence of bullosis diabeticorum--a controversial cause of chronic foot ulceration. International wound journal. 2008;5(4):591-6. Epub 2008/11/14.

21. Riad. H. AH, Mansour. K., Mannai. H., Sada. H., Shaikha. S. Dosari. S. Pruritic Vesicular Eruption on the Lower Legs in a Diabetic Female. Case Report. Case Reports in Dermatological Medicine. 2013;2013(641416):4.

22. Bolognia. L. JJL, Rapini. R. P. Other vesiculobullous diseases. Dermatology2008.

23. Lipsky BA, Baker PD, Ahroni JH. Diabetic bullae: 12 cases of a purportedly rare cutaneous disorder. International journal of dermatology. 2000;39(3):196-200. Epub 2000/04/12.

24. Jung SE, Kim YC. Scleredema of Buschke Following Streptococcal Infection. Annals of dermatology. 2015;27(4):478-80. Epub 2015/08/15.

25. Parmar RC, Bavdekar SB, Bansal S, Doraiswamy A, Khambadkone S. Scleredema adultorum. Journal of postgraduate medicine. 2000;46(2):91-3. Epub 2000/10/03.

26. All MCe. Scleredema diabeticorum in a patient with type 2 diabetes mellitus. Case Rep Endocrinol. 2011;2011:560.
27. Pitarch. G. TA, Martínez-Aparicio. A., Vilata J., Fortea J. Escleredema de Buschke asociado a diabetes mellitus. Estudio de cuatro casos. Actas Dermosifiliogr 2005;96(1):46-9.

28. Kovary PM, Vakilzadeh F, Macher E, Zaun H, Merk H, Goerz G. Monoclonal gammopathy in scleredema. Observations in three cases. Archives of dermatology. 1981;117(9):536-9. Epub 1981/09/01.

29. Besson C. A. KMC, Jacquet. P., Andrac, L., Sayag. J. Electron-beam therapy in scleredema adultorum with associated monoclonal hypergammaglobulinaemia. Br J Dermatol 1994;130(3):394-7.

30. Mattheou-Vakali G, Ioannides D, Thomas T, Lazaridou E, Tsogas P, Minas A Cyclosporine in scleredema. Journal of the American Academy of Dermatology. 1996;35(6):990-1. Epub 1996/12/01.

31. Grundmann-Kollmann M, Ochsendorf F, Zollner TM, Spieth K, Kaufmann R, Podda M. Cream PUVA therapy for scleredema adultorum. Br J Dermatol. 2000;142(5):1058-9. Epub 2000/05/16.

32. Phiske MM. An approach to acanthosis nigricans. Indian dermatology online journal. 2014;5(3):239-49. Epub 2014/08/29.

33. James WD ED, Berger TG. Andrews Diseases of the Skin. Dermatology. C, editor2011. 494-5 p.

34. Judge MR MW, Munro CS. Disorders of keratinization. Burns T BS, Cox N, Griffiths C, editors, editor2010.

35. Hermanns-Le T, Scheen A, Pierard GE. Acanthosis nigricans associated with insulin resistance : pathophysiology and management. American journal of clinical dermatology. 2004;5(3):199-203. Epub 2004/06/10.

36. Tankova T, Koev D, Dakovska L, Kirilov G. Therapeutic approach in insulin resistance with acanthosis nigricans. International journal of clinical practice. 2002;56(8):578-81. Epub 2002/11/12.

37. R.H. Champion JLB, D.A. Burns, S.M. Breathnach. . Metabolic and nutritional disorders, in: Textbook of Derma- tology. Blackwell Science O, UK, editor1998.

38. Bito T, Kawakami C, Shimajiri S, Tokura Y. Generalized eruptive xanthoma with prominent deposition of naked chylomicrons: evidence for chylomicrons as the origin of urate-like crystals. Journal of cutaneous pathology. 2010;37(11):1161-3. Epub 2010/09/21.

39. Parker F. Xanthomas and hyperlipidemias. Journal of the American Academy of Dermatology. 1985;13(1):1-30. Epub 1985/07/01.

40. Behm B, Schreml S, Landthaler M, Babilas P. Skin signs in diabetes mellitus. Journal of the European Academy of Dermatology and Venereology : JEADV. 2012;26(10):1203-11. Epub 2012/02/22.

41. Naik NS. Eruptive xanthomas. Dermatol Online J. 2001;7(2):11. Epub 2002/08/08.

42. Melin H. An Atrophic Circumscribed Skin Lesion in the Lower Extremities of Diabetics. Acta medica Scandinavica. 1964;176:SUPPL 423:1-75. Epub 1964/01/01.

43. Binkley GW. Dermopathy in the diabetic syndrome. Archives of dermatology. 1965;92(6):625-34. Epub 1965/12/01.

44. Vijayasingam SM TA, Chan HL. Non-infective skin associations of diabetes mellitus. Ann Acad Med Singapore. 1988;17:526-35.

45. Lee $\mathrm{CH}$, Tey $\mathrm{BH}$, Chew $\mathrm{W}$. Epidemiology of diabetes mellitus in a regional hospital medical unit. Ann Acad Med Singapore. 1993;22(6):864-9. Epub 1993/11/01.

46. Romano G, Moretti G, Di Benedetto A, Giofre C, Di Cesare E, Russo G, et al Skin lesions in diabetes mellitus: prevalence and clinical correlations. Diabetes research and clinical practice. 1998;39(2):101-6. Epub 1998/05/23.

47. Shemer A, Bergman R, Linn S, Kantor Y, Friedman-Birnbaum R. Diabetic dermopathy and internal complications in diabetes mellitus. International journal of dermatology. 1998;37(2):113-5. Epub 1998/05/16.

48. Morgan AJ, Schwartz RA. Diabetic dermopathy: A subtle sign with grave implications. Journal of the American Academy of Dermatology. 2008;58(3):447-51. Epub 2007/12/25.

49. Chishiki M, Ohnishi Y, Kawada A, Tajima S, Ishibashi A. Diabetic pigmented spots on the extrapretibial region: immunohistochemical study of type IV collagen in dermal vessel wall. European journal of dermatology : EJD. 1998;8(8):560-2. Epub 1999/01/16.

50. Ahmed I, Goldstein B. Diabetes mellitus. Clinics in dermatology 2006;24(4):237-46. Epub 2006/07/11

51. Ngo BT, Hayes KD, DiMiao DJ, Srinivasan SK, Huerter CJ, Rendell MS. Manifestations of cutaneous diabetic microangiopathy. American journal of clinical dermatology. 2005;6(4):225-37. Epub 2005/08/03.

52. Bolognia JL. Jorizzo JL RR. Dermatology Spain e, editor2008.

53. Saray Y, Seckin D, Bilezikci B. Acquired perforating dermatosis: clinicopathological features in twenty-two cases. Journal of the European Academy of Dermatology and Venereology : JEADV. 2006;20(6):679-88. Epub 2006/07/14.

54. AM F. Acquired perforating dermatosis in renal and diabetic patients. . Lancet. 1997;349:895-6. 